Article

\title{
Detailed experiment-theory comparison of mid-infrared metasurface perfect absorbers
}

\author{
Naoki To1 ${ }^{1}$, Saulius Juodkazis ${ }^{2,3,4}$ (D), Yoshiaki Nishijima ${ }^{1,3 *}$ (D) \\ 1 Department of Electrical and Computer Engineering, Graduate School of Engineering, Yokohama National \\ University, 79-5 Tokiwadai, Hodogaya-ku, Yokohama, 240-8501, Japan; nishijima-yoshiaki-sp@ynu.ac.jp \\ 2 Optical Sciences Centre and ARC Training Centre in Surface Engineering for Advanced Materials (SEAM), \\ School of Science, Swinburne University of Technology, Hawthorn, VIC 3122, Australia \\ 3 Institute of Advanced Sciences (IAS), Yokohama National University, 79-5 Tokiwadai, Hodogaya-ku, \\ Yokohama 240-8501, Japan \\ 4 Tokyo Tech World Research Hub Initiative (WRHI), School of Materials and Chemical Technology, Tokyo \\ Institute of Technology, 2-12-1, Ookayama, Meguro-ku, Tokyo 152-8550, Japan \\ * Correspondence:nishijima-yoshiaki-sp@ynu.ac.jp (Y.N.)
}

\begin{abstract}
Realisation of a perfect absorber $A=1$ with transmittance and reflectance $T=R=0$ by a thin metasurface is one of the hot topics in recent nanophotonics prompted by energy harvesting and sensor applications ( $A+R+T=1$ is the energy conservation). Here we tested optical properties of over 400 structures of metal-insulator-metal (MIM) metasurfaces for range of variation in thickness of insulator, diameter of a disc and intra-disc distance experimentally and numerically. Conditions of a near perfect absorption $A>95 \%$ with simultaneously occurring anti-reflection property $(R<5 \%)$ was experimentally determined. Differences between the bulk vs. nano-thin film properties at mid-IR of the used materials can be of interest for plasmonic multi-metal alloys and high entropy metals.
\end{abstract}

Keywords: Metasurface, mid infrared absorption, perfect absorption

\section{Introduction}

Metal-insulator-metal (MIM) type of metasurfaces are used to maximise the absorbance $A$ with no transmittance $T$ and reflectance $R$ at the resonance. In the mid-infrared wavelengths such MIM metasurfaces (Fig. 1) could be designed as the photo-thermal energy converters with luminous IR light converted into heat[1-3]. In our previous work, we have demonstrated the effect of metal and insulator patterns as absorbers and thermal emitters [4]. Heat generation using metasurfaces can provide a mid-IR wavelength source, detectors with narrow bandwidth, and radiative cooling at the atmospheric window wavelengths 3-7 $\mu \mathrm{m}(T \approx 90 \%)$ and $8-13 \mu \mathrm{m}(T \approx 80-60 \%)$. Predictive models of MIMs and other metamaterials with required optical and thermal properties have to be designed based on numerical modeling and optimisation. In this regard the finite-difference time-domain (FDTD) method based on the exact solution of Maxwell's equations is expected to provide the quantitative match to the performance of experimental structures provided material property (electric permitivity $\varepsilon$ ) is known at the used wavelengths. However, we showed recently, that the experimentally made structures have different optical response from the simulated ones due to settle differences in interface roughness and geometry of the nano-pattern [5].

This study is dedicated to address the issue that the maximum absorption $A \rightarrow 1$ is not easily engineered by MIM structures with simultaneously occurring zero reflection condition $R \rightarrow 0$. It is very challenging to realise the perfect absorption condition $A \rightarrow 1$ [5]. A wide parametric study of geometrical characteristics of the MIM patterns is presented to reveal their effect on the optical response 
(a)

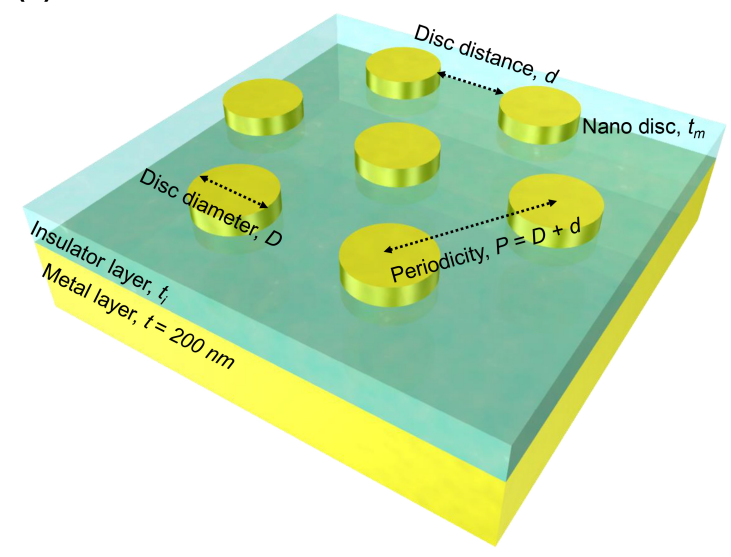

(b)

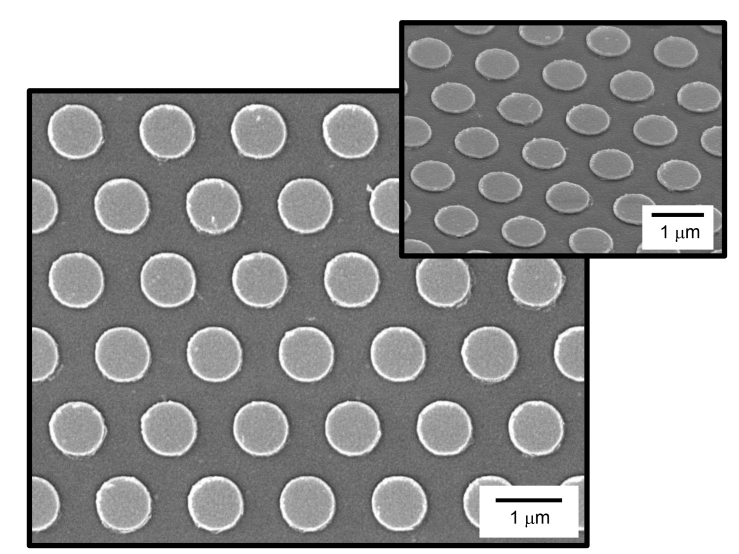

Figure 1. (a)Schematics of the metal-insulator-metal (MIM) metasurface with definition of the geometrical parameters. (b) SEM image of the fabricated metasurface; inset shows a $45^{\circ}$-tilted view SEM image.

of MIM patterns (over 400 samples). In this work we extend the parameter space of metasurfaces and systematically study dependence of $R$ and $A$ on the disc diameters $D$, intra disc separation distance $d$ (the period is $P=D+d$ ), insulator thicknesses $t_{i}$ in the case of fixed MIM materials: $\mathrm{Au}$ film, $\mathrm{SiO}_{2}$, Au nanodisc. Optimisation towards $A=1$ while $R=0$ can be guided by tendencies revealed from the experimental data. All samples (400+) were made during the same material deposition run. This approach inherently takes into account specific properties of thin insulator, metal films and their interfaces dependent on the deposition method, in this case, electron beam (EB) evaporation.

\section{Experiments}

\subsection{Fabrication and characterization of MIM metasurfaces}

Metasurfaces were fabricated with conventional electron beam lithography (EBL) [6]. A 200-nm-thick film of Au has deposited on the glass substrate with a $5 \mathrm{~nm}$ Ti adhesion layer by

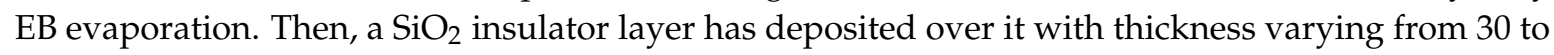
$100 \mathrm{~nm}$ with a $10 \mathrm{~nm}$ increments. After spin coating the EB resist (a twice diluted ZEP $520 \mathrm{~A}$ ), EBL was implemented to draw pattern of circular disks with hexagonal lattice arrangement. Diameter of the disk $D$ was set from 400 to $800 \mathrm{~nm}$ in $100 \mathrm{~nm}$ steps while intra-disc distance $d$ was changing from 100 to $1000 \mathrm{~nm}$. After development of EBL defined disk patterns, a $5 \mathrm{~nm}$ of Ti followed by $50 \mathrm{~nm}$ thickness of Au were deposited. The final step of lift-off in a heated solution of dichloromethane, acetone and methanol was then carried out to obtain the pattern of nano-discs on the top surface.

All the structures were drawn on the same substrate to avoid heterogeneity of the samples (400+) due to fluctuation of the thickness of the first Au film, insulator layer and that of Au nano-discs. This was essential feature of this study which allowed to compare influence of purely geometrical factors of MIM metasurfaces. The reflection spectra of metasurfaces were measured with a micro-spectroscopic Fourier transform FT-IR setup. A $R \sim 98 \%$ reflection Au mirror has used as the references of the reflection spectra. Also we have confirmed a zero transmission with the same FT-IR system. Illustration of the sample geometry and scanning electron microscopy (SEM) images are shown in Fig. 1.

\subsection{FDTD simulations}

The finite-difference time-domain (FDTD) simulations were performed with commercially available software (FDTD solution, Lumerical, Inc.) with three nodes of in-home assembled workstations. The area of simulations was set to the 2.5 periods in $x$-direction and 2.0 periods in 


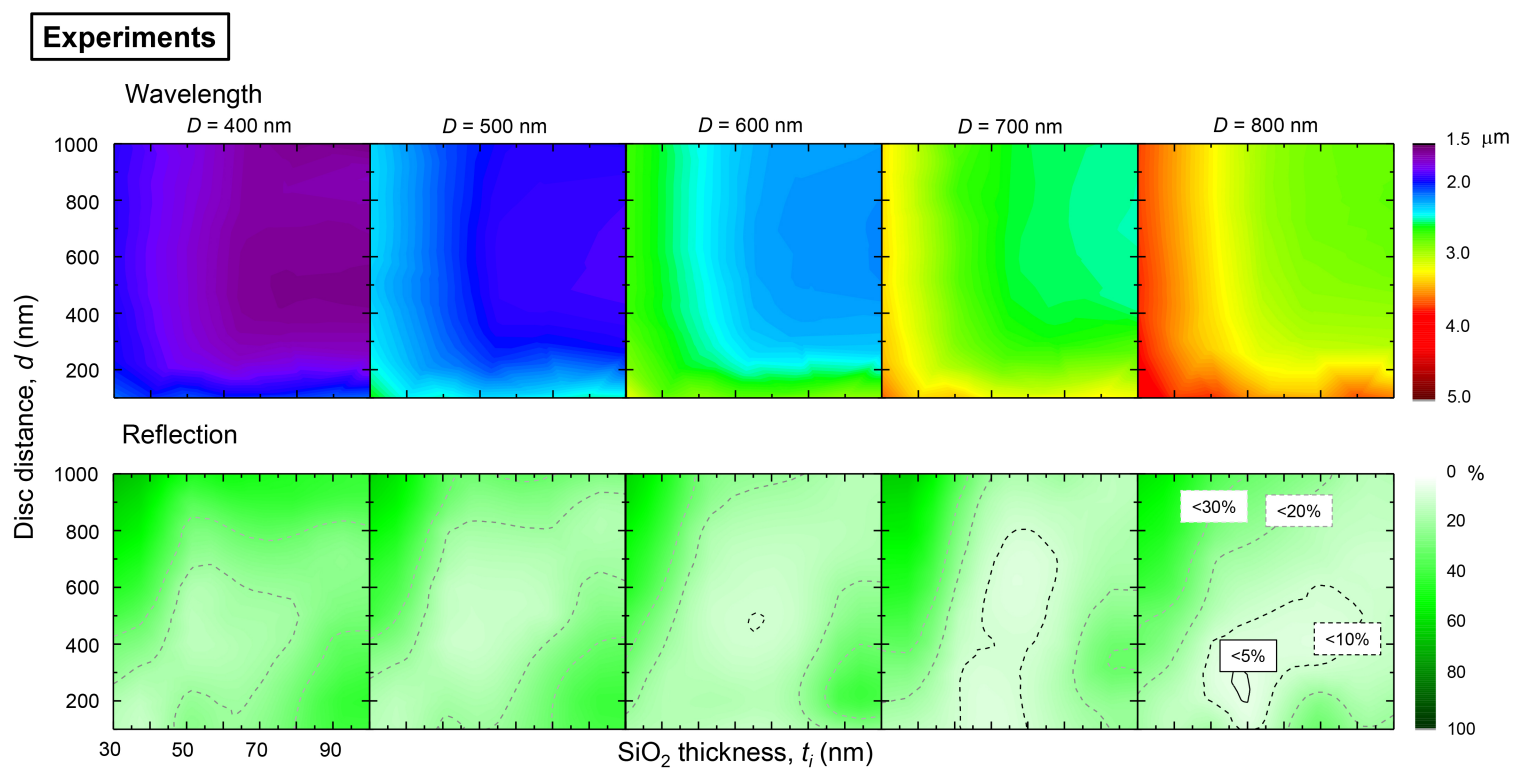

Figure 2. Experimental results of the resonance wavelength (color-coded) and minimum reflectance $R$ obtained from series of reflection spectral measurements $(400+)$ with parameters of disc diameter $D$, intra-disc separation distance $d$ and $\mathrm{SiO}_{2}$ thickness $t_{i}$. Number of samples measured for one $D$ was $(10 \times 8)=80$ for different $t_{i} \times d$.

$\mathrm{y}$-direction for the x-polarised plane wave light source of unit intensity $|E|^{2}=1$. The dielectric material properties, permittivity $\sqrt{\varepsilon}=n+i k$, where $n+i k$ is the complex refractive index taken from the Lumerical database (Palik's spectroscopic reference data[7]) for the mid-IR wavelength region as the default setting.

\section{Results and discussions}

Figure 2 shows experimental results of the resonance wavelength and reflectance at its minimum. The wavelength of the reflectance $R$ minimum is strongly affected by the disc diameter $D$ and $\mathrm{SiO}_{2}$ thickness, but it is almost insensitive to the intra-disc separation when it is larger than $200 \mathrm{~nm}$. When $\mathrm{SiO}_{2}$ layer becomes thicker, the wavelength of the minimum $R$ is blue-shifted and become constant after the specific threshold thickness. The $40 \mathrm{~nm}$ is the characteristic thickness of $\mathrm{SiO}_{2}$ for the $D=400 \mathrm{~nm}$ diameter, but $60 \mathrm{~nm}$ for the $D=800 \mathrm{~nm}$. These tendencies are similar for all disc diameters.

There are two main interactions in nano-disc patterns. First, the interaction of the disk with the metal film. For a thinner $\mathrm{SiO}_{2}$ film, the induced mirror-dipole in the metal film is coupled with the dipole of the plasmon resonance. Second interaction is the dipole-dipole interaction between closest nano-disks. As these dipole-dipole interactions become stronger, the resonance peak wavelength red-shifts. Of course there is anther factor, i.e., a periodic light scattering occurs and affects the plasmon resonance wavelength. The electric field extending from the metal surface into air is strongly wavelength dependent, at a longer wavelength a longer evanescent field extension is observed. Therefore the larger diameter disks, which resonate at the longer wavelengths, have the larger threshold for the separation distance and thickness of insulator layer as compared to the smaller diameters.

These interactions between nano-discs and film as well as with the neighboring discs are also affecting the reflectance. When the intra-disc distance becomes smaller, the reflectance starts decreasing (an increase in optical losses) due to the increased density of nanodiscs (a larger surface area covered by metal). However, the interaction between nano-discs generates a de-localization of the electric field resulting in an increase of the reflectance (a decrease of absorption). This tendency is much clear in the thick $\mathrm{SiO}_{2}$ layers, which have less interaction between mirror dipoles. In case of thinner $\mathrm{SiO}_{2}$, 


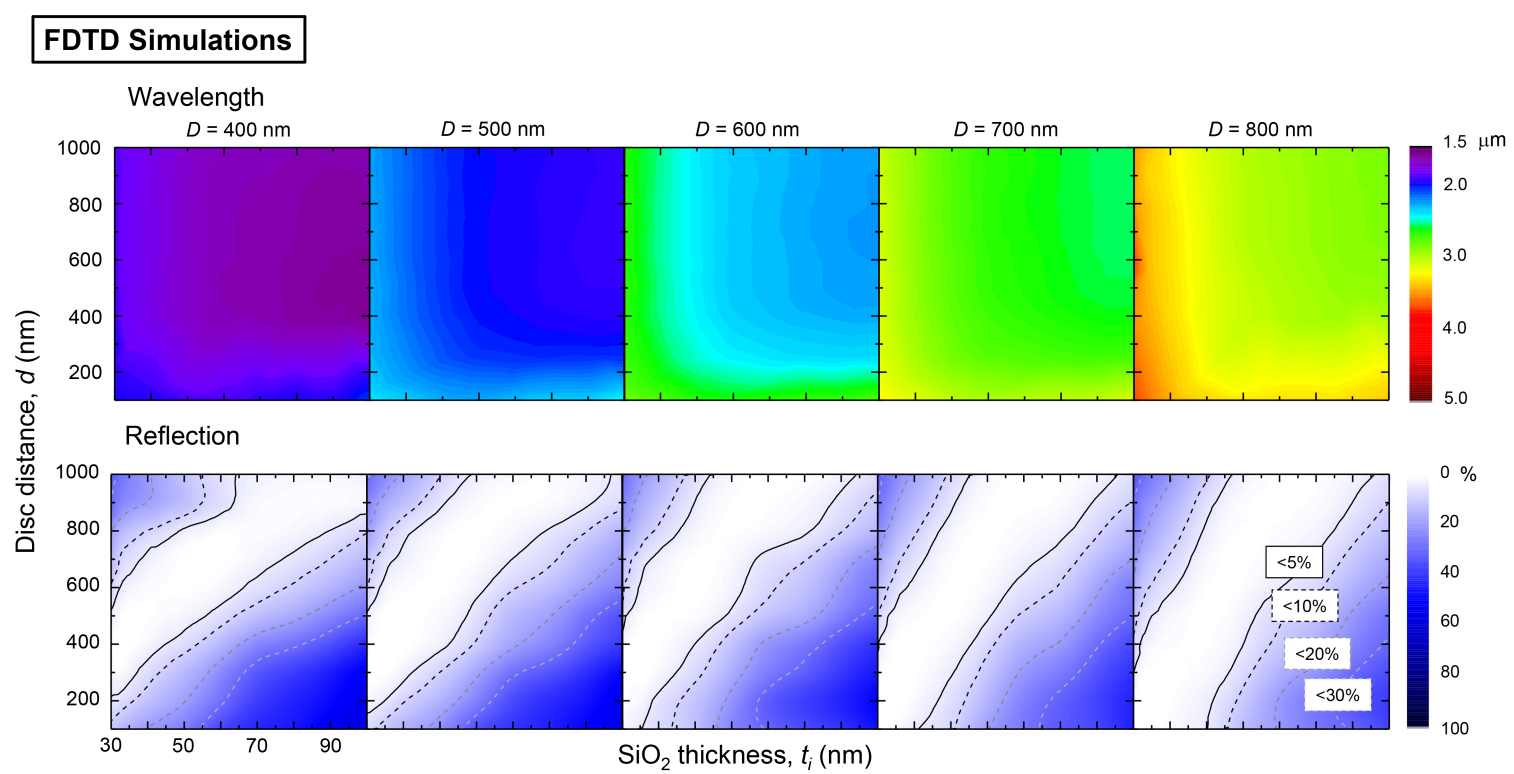

Figure 3. FDTD simulation results of resonance wavelength (color-coded) and minimum reflectance that obtained from series of reflection spectral measurements with parameters of disc diameter $D$, disc distance $d, \mathrm{SiO}_{2}$ thickness $t_{i}$. See Fig. 2 for comparison with experimental results.

reflectance still decreasing until $100 \mathrm{~nm}$ of the intra-disc separation distance $d$. Therefore the color map in Fig. 2 shows tendency of $R$ increase towards the larger $\mathrm{SiO}_{2}$ thickness.

The total mirror dipole interaction can be described by the quadrupole mode that causes a strong electric field localization and larger absorption in the insulator layer. In the series of experimental studies, we found less than the $5 \%$ reflectance condition for the $D=800 \mathrm{~nm}$ discs, $t_{i}=60 \mathrm{~nm} \mathrm{SiO}{ }_{2}$ and $300 \mathrm{~nm}$ intra-disc separation distance.

Figure 3 shows the FDTD simulation results for the same geometry of patterns as in the experimental study of reflectivity. Similar qualitative tendencies of the resonance wavelength are reproduced as in the experiments. However, the reflectance values obtained by FDTD simulations are quite different from the experimental results. Usually, the FDTD simulations show a lower reflectivity at the minimum.

Typically, a commercial FDTD software includes database library of the electric susceptibility (refractive index) for simulations at a specific wavelength range. The used FDTD Lumerical software has a list of popular reference data including, Jhonson and Christy[8], Palik[7], and CRC[9] (C. G. Olson et al., unpublished data, etc.) among others in the database library of dielectric parameters. It is well known that the susceptibility tends to shows different values according to the sample fabrication methods, thickness of the film and analytical methods of characterisation. In Fig. 4, we listed several conventional dielectric parameters from popular data sets by Palik, Mcpeak, Olmon, Werner, Yakbousky, Lermarchand, Jhonson and Christy [7,8,10-14]. These parameters show variation in wavelength and spectral shape. Therefore we analyzed permittivity using the Drude model at longer that $800 \mathrm{~nm}$ wavelength, where it is less affected by the Lorenz part of the permittivity. The Drude equation was used:

$$
\varepsilon(\omega)=\varepsilon_{\infty}-\frac{\omega_{p}^{2}}{\omega^{2}+\Gamma^{2}}+i \frac{\omega_{p}^{2} \Gamma}{\omega\left(\omega^{2}+\Gamma^{2}\right)}
$$

, where $\varepsilon_{\infty}$ is the permittivity at high frequency region, $\omega_{p}$ is the plasma frequency that is related to the free electron density and effective mass of the electron, $\omega_{p}=\sqrt{N_{e}^{2} / \varepsilon_{0} m}$, $\Gamma$ is the damping constant of the free electron oscillation, which is inverse of the relaxation time $\tau$. Using these three parameters as free variables, the permittivity has fitted with least-square method. The result is shown in the Table section of Fig. 4. The $\omega_{p}$ varies only from 1.13 to $1.62 \times 10^{16} \mathrm{~s}$ for different models. However, the $\Gamma$ 


\begin{tabular}{|c|c||c|c|c|}
\hline & & $\omega_{\mathrm{p}} \times 10^{16}\left(\mathrm{~s}^{-1}\right)$ & $\Gamma \times 10^{13}\left(\mathrm{~s}^{-1}\right)$ & $\varepsilon_{\infty}$ \\
\hline \hline (a) & E. D. Palik & 1.13 & 9.11 & -20.8 \\
\hline (b) & K. M. McPeak & 1.41 & 4.91 & 7.64 \\
\hline (c) & R. L. Olmon & 1.24 & 5.76 & -63.7 \\
\hline (d) & W. S. M. Werner & 1.62 & 23.4 & 8.73 \\
\hline (e) & D. I. Yakubovsky & 1.23 & 16.8 & 5.03 \\
\hline (f) & F. Lemarchand & 1.36 & 25.6 & 10.3 \\
\hline (g) & S. Babar & 1.30 & 3.26 & 8.17 \\
\hline (h) & $\begin{array}{c}\text { P. B. Johnson and } \\
\text { R. W. Christy }\end{array}$ & 1.38 & 11.6 & 11.8 \\
\hline
\end{tabular}
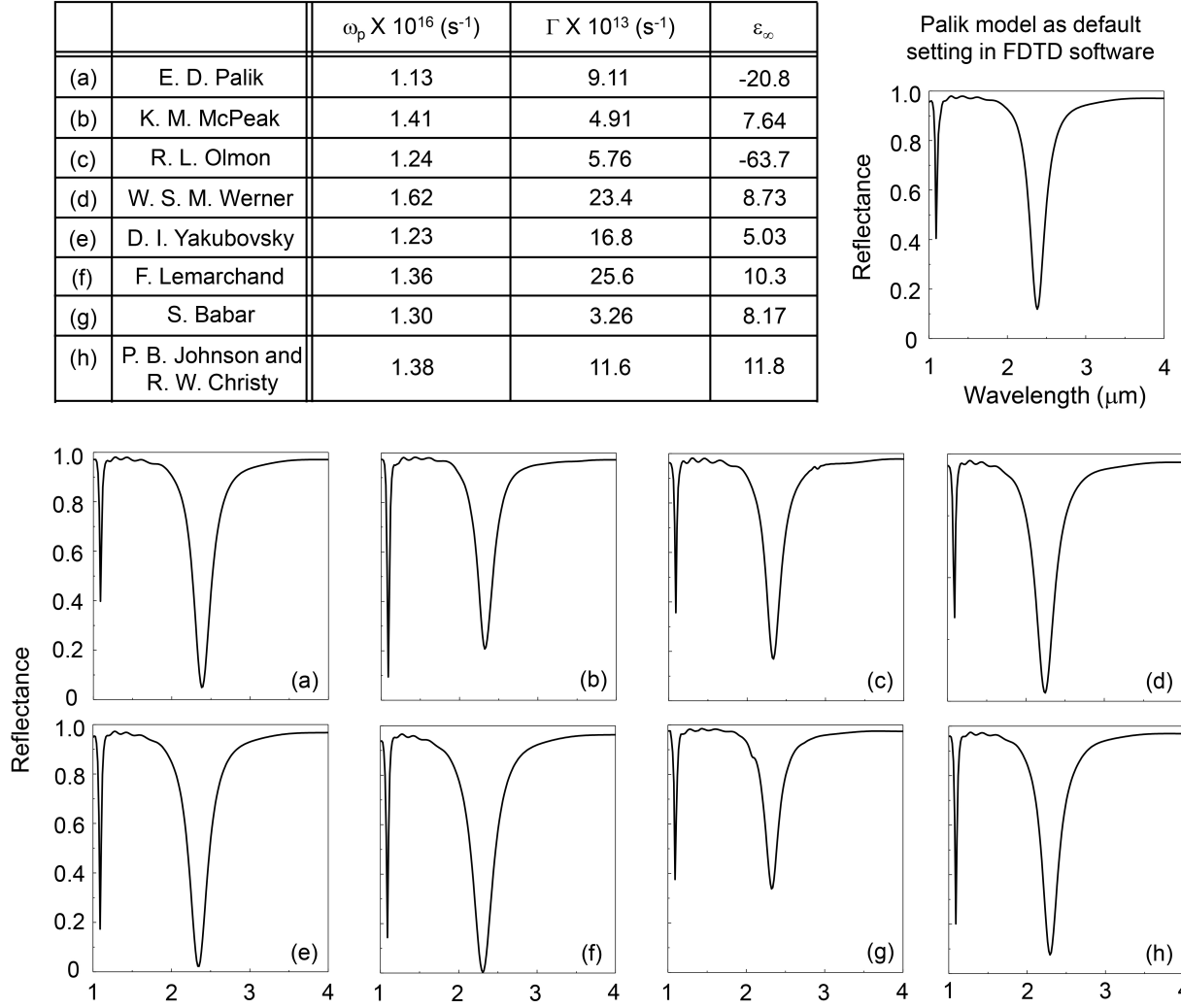

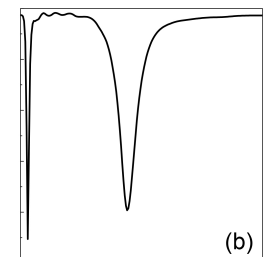

(b)

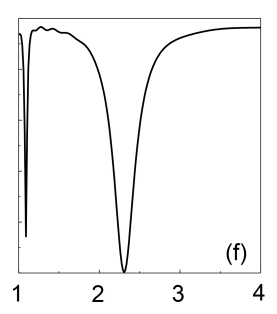

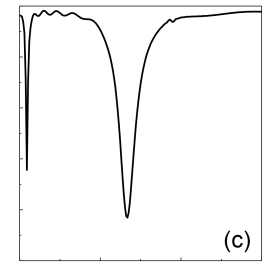

(c)

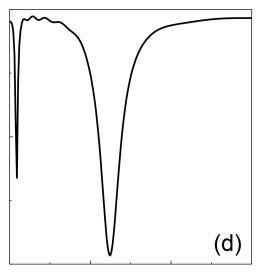

(d)
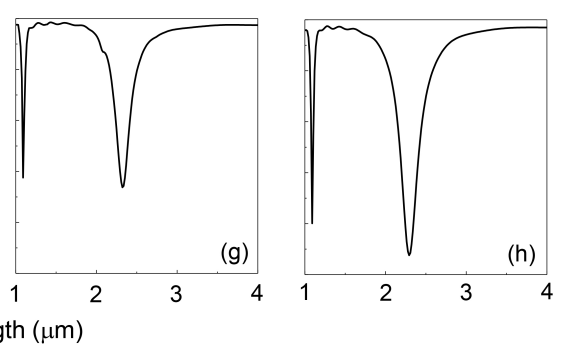

Figure 4. Drude parameters of the permittivity of Au were obtained form the below listed references. The parameters of $D=600 \mathrm{~nm}, P=1200 \mathrm{~nm}, t_{i}=50 \mathrm{~nm}$ were used: (a) Palik[7], (b) McPeak[10], (c) Olmon[11], (d) Werner[12], (e) Yakubovsky[13], (f) Lemarchand[14], (g) Babar[15] and (h) Jhonson and Christy[8]. The FDTD simulation results by (a) Palik parameters from the FDTD Lumerical software database (a default) were used for the Drude model calculations (see Table).

values are spread over a wide range from 3.26 to $23.4 \times 10^{13}$. The $\varepsilon_{\infty}$ is the extrapolated value from the lower frequency region. Therefore, the data have a large fluctuation but it has a minor influence (affect $\sim 1 \%$ ) for the real part of permittivity at mid-IR spectral region. Using these values in the range from 1 to $10 \mu \mathrm{m}$, the values of permittivity were imported into the material database for FDTD simulations (Lumerical). As a default, the Palik model was used in the simulations with results shown in Fig. 2 and 3. The internally fixed parameters (Lumerical) of the tolerance and maximum order coefficients were set to 0.1 and 6 , respectively. Tolerance specifies the target root-mean-square (rms) error between the experimental data and the calculated model. Maximum order coefficients set the maximum number of coefficients allowed in the model. It was found that these values are not completely compatible with the imported permittivity. Due to this difference of the internal parameters, the FDTD simulation results were slightly different from the Drude modeled parameters shown in Fig. 4(a). We set them to 0.01 and 10 to obtain well fitted data within a practically justifiable calculation time. The FDTD simulation results with the structural parameters of $600 \mathrm{~nm}$ diameter disks, $1.2 \mu \mathrm{m}$ period, and $50 \mathrm{~nm}$ of $\mathrm{SiO}_{2}$ thickness were simulated with all nine engineered permittivities. As the reference, we also calculated data according to the Palik model with default settings in Lumerical. The results are shown in Fig. 4.

General tendencies of spectral response obtained form series of FDTD simulations shows that a large $\omega_{p}$ value defines a shorter resonance wavelength. The larger $\Gamma$ defines lower reflectance at the resonance wavelength. The pairs $\omega_{p}$ and reflectivity $R, \Gamma$ and resonance wavelength have no correlation with each other. These tendencies, however, are completely opposite to the experimental measurements 


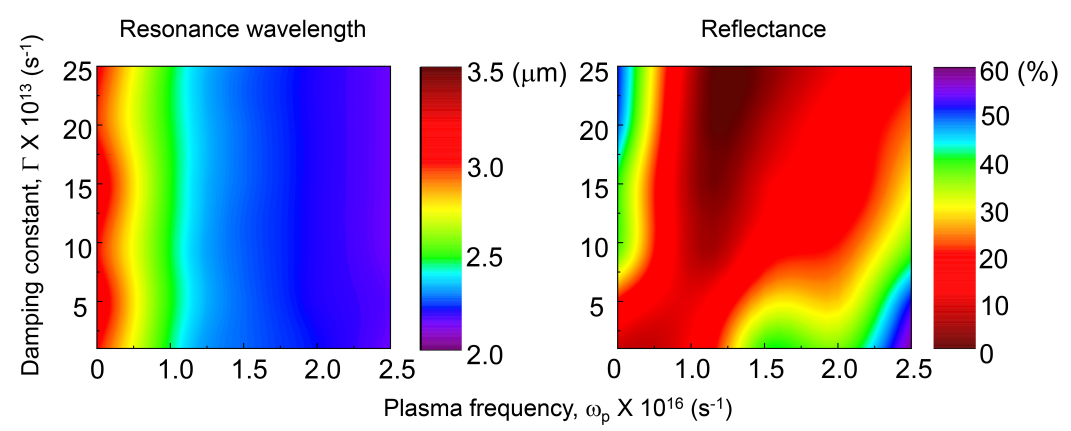

Figure 5. FDTD simulations with artificial Drude parameters that have $\omega_{p}$ from 0.5 to $2.5 \times 10^{16} \mathrm{~s}, \Gamma$ from 1 to $5 \times 10^{13} \mathrm{~s}$ and $\varepsilon_{\infty}=-20.8$ respectively.

of plasmon resonance from nano-disc structures [16-18]. To get insights for the underlying reasons of the experimentally observed tendencies, further FDTD simulations were performed using synthesised permittivities which have $\omega_{p}$ within the 0.5 to $2.5 \times 10^{16} \mathrm{~s}$, $\Gamma$ changing from 1 to $25 \times 10^{13} \mathrm{~s}, \varepsilon_{\infty}=-20.8$ respectively. The bottom metal layer had default Palik's data (Fig. 5).

The results of resonance wavelength and reflectance reproduce well the tendency of experimental results. The sift of the resonance wavelength is related to the interaction between top nano-disc and bottom Au layer. However, the plasma frequency is almost constant because gold has fcc-crystal lattice and the free electron density is fixed by material. Therefore the resonance wavelength obtained in experiment and simulations is the same. On the other hands, $\Gamma$ is related to the electron damping at the grain boundaries, electron-electron and electron-hole scattering. Also, it is related to the relaxation time of the plasmon resonance. At the plasmon resonance, a longer relaxation time is the reason for the higher Q-factor and strong electric field enhancement. However, in the absorptive metasurfaces, a large damping results in an increase of internal absorption due to ohmic losses in the metals. Also, a longer relaxation time might cause an increase of electron scattering influence on the plasmon resonance. Therefore the tendencies observed in experiments are different to the typical plasmon resonance of nano-disc.

The values of $\Gamma$ are affected by the structural quality of films and film deposition methods. The parameter $\Gamma=v_{F} / l$ where $v_{F}$ is the Fermi velocity of electron and $l$ is its mean free path. For the electrons in nanoparticles where the boundaries adds to the scattering when dimensions of the particles are smaller than $l$, the effective mean free path $1 / l^{\prime}=1 / l+a\left(v_{F} / R\right)$, here $R$ is the size of nanoparticle and constant $a \approx 1$ [19]. Ab initio calculations based on electronic band diagram for gold provides $v_{F}=13.82 \times 10^{5} \mathrm{~m} / \mathrm{s}$ and $l=37.7 \mathrm{~nm}$, which can be considerably (more than $30 \%$ ) different from the experimental or model based values [20]. In this study the effect of grain boundaries and film thickness when they are smaller than the free mean path of electron in bulk metal are reflected in the numerical and experimental data.

Absorption and reflectivity are strongly affected by the electron mean free path and contributes to the energy deposition. Usually, the effect of a radiative energy concentration via E-field enhancement due to the large value of real part of refractive index is discussed. Here, we highlight the importance of the imaginary part of the refractive index in energy deposition for design of perfect absorbers $(A \rightarrow 1$ and $R \rightarrow 0$ ). It could be foreseen that optical properties (large $\Gamma$ ) of high entropy metal alloys (with more than 5 constituents) can provide high optical losses and be applied where strong absorbance is required. Black metals with $R \rightarrow 0$ and $T \rightarrow 0$ are made with thin $<10$ nm metal layer in IMI-metasurface [21] and their optical properties are affected by larger losses.

\section{Conclusions and Outlook}

A detailed comparison of experimental and numerical simulation was carried out for MIM metasurfaces to reveal peculiarities how to tune the resonance wavelength and reflectance $R$ of perfect 
absorbers at mid-IR spectral range. Experimental data were collected from over 400 samples which were all made in the same deposition process and only differed by EBL defined sizes of disks and period. All experimental results agreed well with FDTD simulations in the tendency of resonance wavelength and reflectance. FDTD results were strongly affected by the permittivity especially the $\Gamma$ value in the Drude model and thickness of the metal films in MIM metasurface. Therefore, it is important to carefully select the realistic permittivity model or to determine the permittivity of metals experimentally. In series of experiments and simulations, we have revealed that, metal with high ohmic loss metal demonstrates a higher potential to increase the absorption of metasurfaces. Experimentally, less than $5 \%$ reflection was achieved with MIM metasurfaces at specific spectral windows. This constitute an engineering art to achieve $A \rightarrow 1$ in $A+R+T=1$.

The explored absorbance enhancement at the mid-IR spectral window is important for number of bio-medical and environmental sensor applications, e.g., biological $\mathrm{N}_{2}$ fixation is the determining factor in $\mathrm{CO}_{2}$ uptake by plants from atmosphere [22]. The atmospheric transmission window with $T \approx 90 \%$ between 3 and $4 \mu \mathrm{m}$ wavelengths can be utilised for radiative cooling. The Boltzmann temperature of $3.5 \mu \mathrm{m}$ radiation is $T=h v / k_{B}=4.11 \times 10^{3} \mathrm{~K}$ defining the limit of the heat to work conversion in Carnot engine, here $h v$ is the photon energy and $k_{B}$ is the Boltzmann constant. Structures and surfaces for high reflectivity or transmissivity at 1-4 $\mu \mathrm{m}$ IR wavelengths can be made by 3D laser polymerisation with direct writing or holographic recording [23-25]. Surfaces designed for enhanced absorption can be used as efficient radiators at the same wavelengths according to the Kirchhoff's principle demonstrated experimentally at IR spectral range [4].

Author Contributions: conceptualization, Y. N., N. T. and S. J.; methodology, Y. N., N.T.; validation, N. T.; formal analysis, Y. N.; investigation Y.N., N.T.; data curation, Y. N., N. T.; writing-original draft preparation, Y. N.; writing-review and editing, all authors; project administration, Y. N.; funding acquisition, Y. N., S. J.

Funding: This research was funded by Japan Society for the Promotion of Science (JSPS), Grants-in-Aid for Scientific Research, JSPS Bilateral Joint Research Projects between Japan and Lithuania JSPSBP120194203. SJ acknowledges partial support by the ARC Discovery DP190103284, Linkage LP190100505 and JST CREST JPMJCR19I3 grants.

Conflicts of Interest: The authors declare no conflict of interest.

1. Lochbaum, A.; Fedoryshyn, Y.; Dorodnyy, A.; Koch, U.; Hafner, C.; Leuthold, J. On-Chip Narrowband Thermal Emitter for Mid-IR Optical Gas Sensing. ACS Photonics 2017, 4, 1371-1380.

2. Tsai, M.W.; Chuang, T.H.; Meng, C.Y.; Chang, Y.T.; Lee, S.C. High performance midinfrared narrow-band plasmonic thermal emitter. Applied Physics Letters 2006, 89, 2-5.

3. Yang, Z.Y.; Ishii, S.; Yokoyama, T.; Dao, T.D.; Sun, M.G.; Pankin, P.S.; Timofeev, I.V.; Nagao, T.; Chen, K.P. Narrowband wavelength selective thermal emitters by confined tamm plasmon polaritons. ACS Photonics 2017, 4, 2212-2219.

4. Nishijima, Y.; Balčytis, A.; Naganuma, S.; Seniutinas, G.; Juodkazis, S. Kirchhoff's metasurfaces towards efficient photo-thermal energy conversio. Sci. Reports 2019, 9, 8284.

5. Nishijima, Y.; Balčytis, A.; Naganuma, S.; Seniutinas, G.; Juodkazis, S. Tailoring metal and insulator contributions in plasmonic perfect absorber metasurfaces. ACS Appl. Nano Mater. 2018, 1, 3557-3564.

6. Beni, T.; Yamasaku, N.; Kurotsu, T.; To, N.; Okazaki, S.; Arakawa, T.; Balčytis, A.; Seniutinas, G.; Juodkazis, S.; Nishijima, Y. Metamaterial for Hydrogen Sensing. ACS Sens. 2019, 4, 2389-2394.

7. Palik, E.D. Handbook of optical constants of solids; Academic Press, INC., Washington, D. C., United states, 1985; pp. 286-295.

8. Johnson, P.B.; Christy, R.W. Optical constants of the noble metals. Phys. Rev. B 1972, 6, 4370-4379.

9. Lide, D.R. CRC handbook of chemistry and physics; Vol. 73, CRC Press, INC., United states, 1985; pp. 12 111-114.

10. McPeak, K.M.; Jayanti, S.V.; Kress, S.J.P.; Meyer, S.; Iotti, S.; Rossinelli, A.; Norris, D.J. Plasmonic films can easily be better: Rules and recipes. ACS Photon. 2015, 2, 326-333. 
11. Olmon, R.L.; Slovick, B.; Johnson, T.W.; Shelton, D.; Oh, S.H.; Boreman, G.D.; Raschke, M.B. Optical dielectric function of gold. Phys. Rev. B 2012, 86, 235147.

12. Werner, W.S.M.; Glantschnig, K.; Ambrosch-Draxl, C. Optical constants and inelastic electron-scattering data for 17 elemental metals. J. Phys Chem Ref. Data 2009, 38, 1013-1092.

13. Yakubovsky, D.I.; Stebunov, Y.V.; Kirtaev, R.V.; Ermolaev, G.A.; Mironov, M.S.; Novikov, S.M.; Arsenin, A.V.; Volkov, V.S. Ultrathin and ultrasmooth gold films on monolayer $\mathrm{MoS}_{2}$. Adv. Mater. Interfaces 2019, 6, 1900196.

14. Gao, L.; Lemarchand, F.; Lequime, M. Comparison of different dispersion models for single layer optical thin film index determination. Thin Solid Films 2011, 520, 501-509.

15. Babar, S.; Weaver, J.H. Optical constants of Cu, Ag, and Au revisited. Appl. Opt. 2015, 54, 477-481.

16. Hashimoto, Y.; Seniutinas, G.; Balčytis, A.; Juodkazis, S.; Nishijima, Y. Au-Ag-Cu nano-alloys: tailoring of permittivity. Sci. Reports 2016, 2, 25010.

17. Nishijima, Y.; Hashimoto, Y.; Seniutinas, G.; Rosa, L.; Juodkazis, S. Engineering gold alloys for plasmonics. Appl. Phys. A 2014, 117, 641-645.

18. Nishijima, Y.; Akiyama, S. Unusual optical properties of the Au/Ag alloy at the matching mole fraction. Opt. Mater. Express 2012, 2, 1226-1235.

19. Cai, W.; Shalaev, V. Optical metamaterials; Springer, New York, 2010.

20. Gall, D. Electron mean free path in elemental metals. J. Appl. Phys. 2016, 119, 085101.

21. Lundgaard, S.; Ng, S.; Nishijima, Y.; Mazilu, M.; Juodkazis, S. Black Metals: Optical Absorbers. Micromachines 2020, 11, 256.

22. Stockmann, U.; Adams, M.A.; Crawford, J.W.; Field, D.J.; Henakaarchchi, N.; Jenkins, M.; Minasny, B.; McBratney, A.B.; Courcelles, V.; Singh, K.; Wheeler, I.; Abbott, L.; Angers, D.A.; Baldock, J.; Bird, M.; Brookes, P.C.; Chenu, C.; Jastrow, J.D.; Lal, R.; Lehmann, J.; O’Donnell, A.G.; Parton, W.J.; Whitehead, D.; Zimmermann, M. The knowns, known unknowns and unknowns of sequestration of soil organic carbon. Agricul. Ecosyst. Environ. 2013, 164, 80-99.

23. Seet, K.; Mizeikis, V.; Juodkazis, S.; Misawa, H. Three-dimensional horizontal circular spiral photonic crystals with stop gaps below 1 micrometer. Appl. Phys. Lett. 2006, 88, 221101.

24. Kondo, T.; Juodkazis, S.; Mizeikis, V.; Matsuo, S.; Misawa, H. Fabrication of three-dimensional periodic microstructures in photoresist SU-8 by phase-controlled holographic lithography. New J. Phys. 2006, 8, 250.

25. Žukauskas, A.; Malinauskas, M.; Kadys, A.; Gervinskas, G.; Seniutinas, G.; Kandasamy, S.; Juodkazis, S. Black silicon: substrate for laser 3D micro/nano-polymerization. Opt. Express 2013, 21, 6901-6909. 